\title{
El signo de Einstein y otros signos de aneurisma de aorta
}

\author{
P. YOUNG, B. C. FINN, D. PELLEGRINI \\ Servicio de Clínica Médica. Hospital Británico. Buenos Aires. Argentina
}

\begin{abstract}
RESUMEN
Se conoce como signo de Einstein a un cuadro similar a la colecistitis aguda causado por la ruptura de un aneurisma de aorta abdominal. En este artículo describimos la evolución de la enfermedad que padeció el renombrado físico hasta llevarlo a la muerte, realizando posteriormente una revisión de otros signos que pueden hallarse en algunos pacientes con aneurisma de aorta, ruptura o disección.
\end{abstract}

PALABRAS CLAVE: Aneurisma de aorta. Signo de Einstein.

\author{
EINSTEIN'S SIGN AND OTHER SIGNS OF AORTIC ANEURYSM
}

\begin{abstract}
Einstein's sign is a syndrome similar to an acute cholecystitis caused by the rupture of an abdominal aortic aneurysm. In this paper we describe the evolution of the disease that the physicist suffered till it led him to death. We also revise other signs which could be found in some patients with ruptured or dissected abdominal aortic aneurysm.
\end{abstract}

KEY WORDS: Aortic aneurysm. Einstein's sign.

Young P, Finn BC, Pellegrini D. El signo de Einstein y otros signos de aneurisma de aorta. An Med Interna (Madrid) 2006; $23: 345-346$.

Después de 29 años de la muerte de Albert Einstein, un médico del mismo hospital en el cual murió, en Princeton (Nueva Jersey, EE.UU.), bautizó con su nombre a un cuadro similar a la colecistitis aguda ocasionado por la ruptura de un aneurisma de aorta abdominal (1-3).

Varios años antes de su muerte, debido a las molestias abdominales que el físico padecía, aconsejado por sus médicos y amigos Rudolf Ehrnann y Gustav Bucky, decidió someterse a una laparotomía exploradora el 31 de diciembre de 1948, en el hospital judío de Brooklyn, Nueva York.

En aquella cirugía realizada por el renombrado cirujano Rudolf Nissen, se encontró un aneurisma calcificado del tamaño de un pomelo en la porción final de la aorta. En aquellos tiempos no era posible su reparación, por lo cual se cubrió con celofán y se realizó una omentopexia. Permaneció relativamente asintomático por un periodo de 6 años y medio, hasta que el 10 de abril de 1955 comenzó a experimentar vagos dolores abdominales. Tres días después éstos se hicieron más fuertes, agregándose náuseas y vómitos. El doctor GK Dean, médico de cabecera, notó un crecimiento del aneurisma y diagnosticó una posible fisura del mismo. Horas más tarde el Dr. Dean discutió el caso con Ehrnann y Bucky, quienes sospecharon una colecistitis aguda. Al día siguiente, 14 de abril, al empeorar los dolores fue llamado como consultor el doctor Franz Glenn, cirujano cardiovascular de Nueva York, tras revisarlo, propuso la intervención dada la sospecha de colecistitis y una grieta en el aneurisma. Al no brindarle garantías sobre el resultado de esta, Einstein la rechazó. Posteriormente afirmaría: "Quiero marcharme cuando yo quiera, es de mal gusto prolongar la vida artificialmente; ya hice mi parte, y es hora de marcharme. Lo haré con elegancia".

Como los dolores no cesaban, siendo cada vez más intensos, y al sumarse ahora signos de deshidratación, fue internado en el Hospital de Princeton. En ese momento, el dolor se localizaba en el hipocondrio derecho y era muy evidente el latido del aneurisma.

A pesar de los analgésicos persistía el dolor, lo que no impidió que Einstein recibiera visitas, trabajara en sus cálculos y en un discurso pacifista que estaba redactando en colaboración con Bertrand Russell. 
El día 17 de abril el médico de cabecera, en su última visita a las 23 horas, comprobó que dormía. Dos horas más tarde, tras murmurar unas palabras en alemán, Einstein de 76 años de edad falleció a la 1,15 hora del 18 de abril de 1955. El doctor Thomas Harvey, patólogo forense que realizó la autopsia, constató que la muerte se debió a una hemorragia por rotura aneurismática. La vesícula estaba intacta.

Así como el signo de Einstein, se pueden encontrar ciertos hallazgos en el examen físico en algunos pacientes con aneurisma de aorta, disección o ruptura. Entre ellos recordamos los siguientes, por orden alfabético:

Signo de Bozzolo Nicoladone: vasos pulsátiles en la mucosa nasal, que pueden aparecer en el aneurisma torácico.

Signo de Cardarelli: al llevar la tráquea lateralmente hacia un lado y luego hacia el otro del cuello, se aprecian pulsaciones sincrónicas con el pulso (Cardarelli derechoizquierdo).

Signo de Corrigan: pulsación peculiar de manera ampliada en abdomen que indica aneurisma de aorta abdominal.

Signo de Dorendorf: plenitud en el hueco supraclavicular en el aneurisma del cayado.

Signo de Drucmond: soplo que se escucha con la boca abierta durante la respiración, en caso de aneurisma del cayado aórtico.

Signo de Glasgow: soplo sistólico que se percibe sobre la arteria braquial en el aneurisma de aorta.

Signo de Hall: choque traqueal diastólico perceptible en el aneurisma de aorta.

Signo de Hope: doble latido cardíaco en caso de aneurisma de aorta.

Signo de Jaccoud: prominencia de la aorta en la escotadura supraesternal.

Signo de Logue: pulsación de la articulación esternoclavicular en el aneurisma del cayado aórtico.

Signo de Oliver: al tomar el cartílago cricoides entre los dedos pulgar e índice y elevarlo, se aprecia un tironeo rítmico coincidente con cada contracción cardíaca.

Signo de Pérez: estertor audible sobre la parte superior del tórax cuando los brazos se levantan y se bajan sucesivamente, fenómeno común en casos de mediastinitis fibrosa y del aneurisma del cayado aórtico.

A través de la historia, los médicos se han dedicado a encontrar pistas que conduzcan a un diagnóstico correcto en el caso de una patología tan delicada como la aórtica.

En el mundo de la medicina de hoy, donde la tecnología juega un rol importante, como dice Alberto Agrest (4): "hemos llegado a escuchar poco, explicar menos, pedir muchos exámenes auxiliares y decidir sobre la base de lo que los exámenes nos informan", perdiendo así el valor relevante de la anamnesis y el examen físico.

\section{Bibliografía}

1. Chandler JJ. The Einstein sign: the clinical picture of acute cholecystitis caused by ruptured abdominal aortic aneurysm. N Engl J Med 1984; 310: 1538.

2. Cohen JR, Graver LM. The ruptured abdominal aortic aneurysm of Albert Einstein. Surg Gynecol Obstet 1990; 170: 455-8.

3. Montes Santiago J. El signo de Einstein en la rotura de un aneurisma

abdominal. Rev Clin Esp 2002; 202: 624.

4. Agrest A. Pasado, presente y futuro en la enseñanza de la medicina clínica. In: Cámera MI, Romani A, Madoery C, Farías J, editors. Avances en medicina '94. Buenos Aires: Sociedad Argentina de Medicina; 1994, p 295-306. 